\title{
Towards a Strategic Model for Safeguarding the Preservation of Business Value During Human Interactions with Information Systems
}

\author{
Chris D. Grobler and Thomas M. van der Merwe ${ }^{(\bowtie)}(\mathbb{D}$ \\ School of Computing, University of South Africa, Science Campus, \\ 28 Pioneer Avenue, Florida Park, Roodepoort 1709, South Africa \\ cdg.postman@outlook.com,vdmertm@unisa.ac.za
}

\begin{abstract}
This paper considers the dichotomy inherent in Information Systems where its introduction, for the purposes of creating new or sustaining existing business value, subsequently also inadvertently or deliberately dissipates value. We investigate root people-induced causes, delineated within a rudimentary Conceptual Technology Value Framework. To support a qualitative investigation, the framework is forthwith applied as the basis for a series of interviews within a major South African financial institution operating within the disciplines of information technology, business operations and organisational development. The constructs identified are discussed and find gestalt in an Adjusted Technology Value Model which can be used to safeguard business value against destructive HCI behaviors.
\end{abstract}

Keywords: Information systems $\cdot$ Business value $\cdot$ Value creation $\cdot$ Value Dissipation $\cdot$ Human computer interaction

\section{Introduction}

A topic that has been debated for many years without a clear resolution is the dualistic nature of an information system (IS) impacting on business value, where the adoption and use of an IS in an organisation as an explicit value creator, also brings about the destruction of business value [1]. In adopting a slightly dystopic view, our focus in this paper is seated within the context of the potentially negative impact that end-users have on organisations when discontinuing the use of a particular mandated IS [2], or making misuse of information within an IS that is intended to drive value realisation [3, 4].

Of the numerous studies [e.g. 5, 6] that have placed specific focus on the interactive relationships between humans and computers (HCI) and endeavoured to explain how these relationships contribute positively towards organisational objectives, none have attempted to expressly illuminate the phenomenon where human agents erode information technology enabled benefits through the consumption of IS. Although the primary cause of organisational value erosion was identified by several authors [e.g. 7, 8] to be the human agent, again, none of these authors attempted to articulate the actual IS related behavioural activities or actions executed by the human agent contributing directly to business or organisational value erosion. This is the issue that this paper seeks to investigate. 
The primary research question this paper asks is: How can business value be safeguarded against destructive HCI behaviors? The primary purpose is to build a value framework from which an empirically-endorsed model can be constructed, and through which the unintended business value dissipating effects on institutions, as a direct result of end-user's misuse of IS, may be investigated and moderated.

Three secondary objectives that dictate the structure of this paper are pursued: (1) to review key characteristics from several germane models and theories relating to the business impact of HCI that maps to, and refines, a rudimentary Conceptual Technology Value Framework (CTVF), (2) to apply the CTVF as a basis for a qualitative investigation from which an Adjusted Technology Value Model (ATVM) may be derived and contextualized, and (3) to present the ATVM as a first benchmark to identify, investigate, mitigate and minimise or eliminate unintentional value destroying effects.

\section{Background}

In construction of our CTVF, key characteristics from selected germane models and theories relating to the negative business impact of HCI as they relate to our own professional experiences as end-users and IS practitioners were considered. Here it is noted that the relationship between IS use and performance is complex and therefore invites multiple theoretical approaches. Our approach is mainly inductive i.e. the CTVF we propose is not intended to be fully grounded in the literature. We simply chose to follow a bottom-up approach while maintaining a minimum level of theoretical sensitivity. An extensive review of the literature yielded the following three models that contained elements of both HCI and delinquent employee behavior which in turn provided the conceptual constructs framing the CTVF delineated in Fig. 1.

Task-Technology Fit (TTF): The model [9] asserts that for technology to have a positive impact on a user's performance, IS user utilisation is required, while an alignment between the characteristics of the task that the user must perform, and the technology needs to exist. In our view, the TTF supports a phenomenon where users may unintentionally misuse an IS if some form of misalignment between the user, enabling technology and the task that the user must perform, exists.

Lazy User Theory (LUT): The theory [10] moves from the premise that in fulfilling a user need, he will be biased towards those solutions that are perceived as most suitable and usable based on the lowest level of effort. The LUT presents a theoretical situation where a user, partial to a legacy IS, will favour the use of said system above that of a newly introduced system, thereby passively disusing the new system, regardless of its utility.

Agency Theory (AT): When a self-interested (agent) individual is requested (by a principal) to perform a specific task, he will be motivated by three different conditions: He will perform the task because he is forced to, or he knows he must, or he wants to [11]. AT potentially considers the phenomenon where a self-interested employee will endeavour to actively abuse the company's IS for personal gain, and/or, in extreme 
cases, intentionally sabotage the assets of an organisation to achieve some selfinterested objective.

From the succinct review of the model/theories it is evident that the benefits realisation of IS usage is fundamentally informed by the actions and behaviours of individuals within the organisational context. Within the context of systems thinking, the structure of a system is constituted by the systemic interrelationships between feedback loops, concluding that said structure constitute the primary driver for a system's behaviour [12]. Within our CTVF, two feedback loops are proposed. The degree of control loop attempts to control both quiescent and recalcitrant user behaviour during system usage, while the degree of influence loop endeavours to influence user beliefs, attitudes, and intention, towards correct and optimal system use.

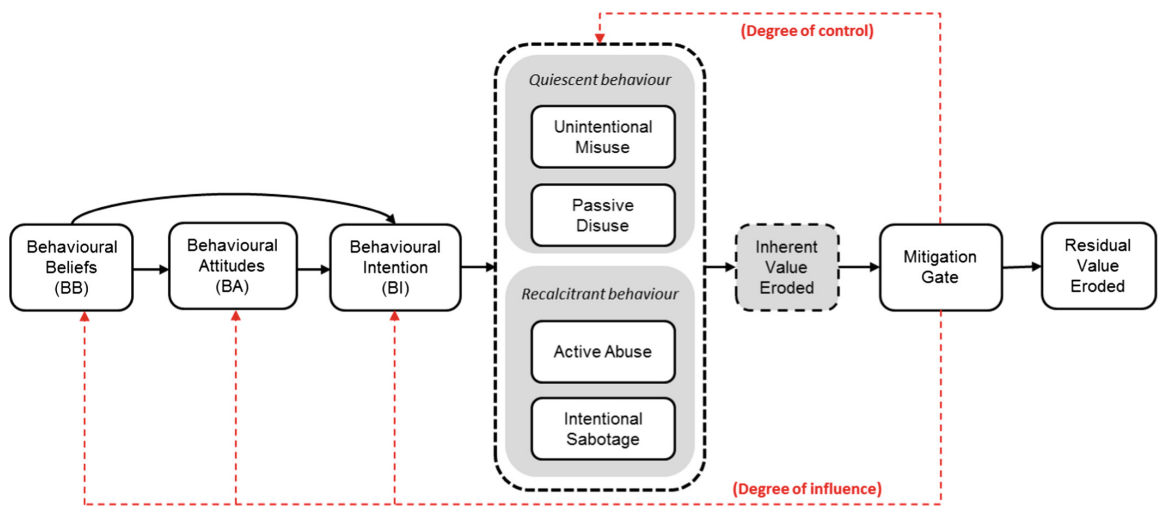

Fig. 1. Conceptual technology value framework

In Fig. 1, moving from left to right, the CTVF constructs are described as follows: behavioural beliefs, behavioural attitude and behavioural intention will be applied as in the Wixom and Todd Research Model [13], the latter which ties constructs from the user satisfaction and technology acceptance literature into a single research model. Next, unintentional misuse and passive disuse are both assumed to possess quiescent qualities. The unintentional misuse construct denotes actual behaviour where the user is misapplying the system, either consciously or unconsciously, due to a lack of skill or negligence. In contrast, passive disuse can be described as a user's passive-aggressive attitude towards having to use a system, causing the user to avoid interaction with said system. The two recalcitrant value eroding behaviour constructs describe a more sinister scenario. Active abuse encompasses situations where a user determinedly employs the system for personal gain or to perform unauthorised transactions. Finally, intentional sabotage designates the purposeful disruption or damage to a system by a disgruntled user. The outcomes of each of the actual value eroding behaviour constructs is summated into the inherent value eroded determinate which is a precursor to the mitigation gate. The latter mediates between the inherent value eroded and the residual value eroded as it attempts to moderate undesirable actioned behaviour 
through system controls and human influence. The final construct of residual value eroded defines the latent value eroded after measures had been taken to reduce the value erosive effects caused by system users.

\section{Methodology and Results of Metadata Analysis}

The data collection process comprised semi-structured interviews with a convenience sample of 31 professional and experienced employees at a major South African financial institution operating within the disciplines of information technology, business operations and organisational development. Semi-structured interviews required participants to deliberate on the CTVF constructs, their validity, significance, rankings, interrelationship and impact management. A descriptive content analysis method [14] was applied to extract themes and contradictions within the data.

Table 1 presents the results of our metadata analysis. It is evident that, on average, the participants used phrases and terms specific to the four value-eroding behaviours most, followed by the three Behavioural Constructs and the four factors comprising the mitigation of value erosion. The concept of Value Dissipation also scored highly, as did various behavioural relationships. Value Eroding Potential returned mid-range totals. By and large, participants concurred with the statement that the introduction of IS may not only create value for, but likewise inadvertently dissipate value from organisations. Participants also consistently referred to value erosion as a by-product of value creation especially within the areas of unintentional misuse and passive disuse.

Table 1. Most mentioned aspects by 31 participants

\begin{tabular}{|c|c|c|c|c|c|c|c|c|c|c|c|c|c|c|c|}
\hline 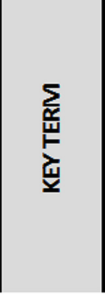 & 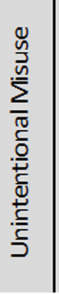 & 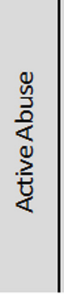 & 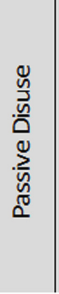 & 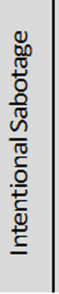 & 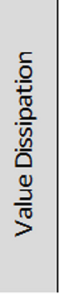 & 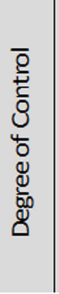 & 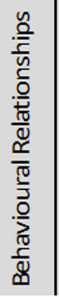 & 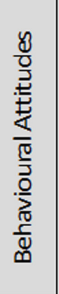 & 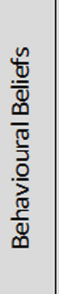 & 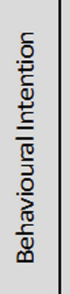 & 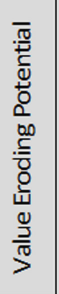 & 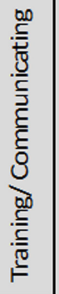 & 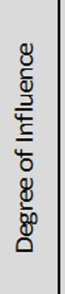 & 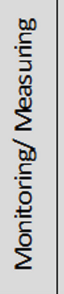 & 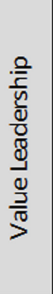 \\
\hline & 220 & 200 & 197 & 194 & 168 & 164 & 150 & 141 & 130 & 130 & 124 & 99 & 75 & 75 & 15 \\
\hline$\overline{V E}$ & 7.1 & 6.5 & 6.4 & 6.3 & 5.4 & 5.3 & 4.8 & 4.5 & 4.2 & 4.2 & 4.0 & 3.2 & 2.4 & 2.4 & 2.4 \\
\hline
\end{tabular}

\section{Findings}

\subsection{Behaviour Constructs Themes}

Unintentional Misuse: Users may simply not be aware that they are engaging unintentional misuse: “...if you don't know what you're doing is wrong, it means in your 
mind what you're doing is correct and it's appropriate. So it goes hand in hand with unintentional misuse". (Participant 09). Unintentional misuse was extended to cases where individuals do not make optimal use of a system e.g. front-line completing only mandatory fields, with the role of management in correcting unintentional misuse considered important.

Passive Disuse: Passive disuse is perceived to be destroying value: “...that destroys value because, immediately what you have is, you have double work and you also have something that you've paid for that's not being used, so you're effectively wasting a license. So, that is definitely also dissipating value". (Participant 05). A shared consensus prevailed that passive disuse introduced numerous instances of complexity and undesirable noise into the overall IS landscape with time pressure identified as a contributing factor to users returning to familiar legacy systems. Individual passive disuse is also viewed a precursor to team passive disuse, where individuals rationalise improper behaviour and ultimately tend to share workarounds with their colleagues. System controls and managerial superintendence were identified as the most effective counter measures.

Active Abuse: Except for agreement on the pervasive nature of active abuse, participants did not agree on the extent to which active abuse eroded business value. Active abuse and intentional sabotage were perceived to be reinforcing constructs that: “... feed each other". (Participant 23). Some users may perceive themselves to be selfappointed end-user testers of production systems, and through actions of unsolicited active abuse create awareness of weaknesses and inefficiencies in a particular IS.

Intentional Sabotage: No evident pattern emerged. Despite the improbability of intentional sabotage, many agreed that it could possibly cause the greatest harm. One participant noted that a user's deviant belief system developed: “... when people's behavioural beliefs don't align with the values of the organisation”. (Participant 27). Two participants highlighted the possibility that some users may be sabotaging systems with good intent, i.e. to draw attention to problems embedded in systems.

Interrelationship Between the Four Constructs: While to most participants the relationships between respectively the two quiescent behaviours and the two recalcitrant behaviours were clear, not all agreed on the existence of potential relationships crossing over between quiescent and recalcitrant constructs.

\subsection{Mitigating Constructs Themes}

Degree of Control: Participants described control measures as being useful in the prevention, detection and correction of undesirable behaviour, but of little value in addressing individuals' beliefs, attitudes and intentions. Preventative controls were perceived to be more desirous as well as managerial oversight and the examples they set.

Degree of Influence: Of paramount importance, executive leadership should positively influence the moral values of employees, to cascade down to every end-user, and 
which will marginalise individuals with corrupted belief systems, attitudinal problems or malicious intention. "The technology is important but without the users to drive the systems, and effectively leaders to guide the users, the unfortunate outcome would be a failed IS”. (Interviewee 10).

\section{Discussion and Adjusted Technology Value Model}

While all the framework constructs were qualitatively endorsed, various arguments exist for, and against relationships, or not. Comparing the CTVF (Fig. 1) to the proposed ATVM (Fig. 2), it is evident that participants were not in agreement as to the flow of the former, the most common view suggesting that while there appears to be a tendency for Behavioural Beliefs and Behavioural Attitudes to display a closer relationship with the quiescent behaviours, and Behavioural Intention, in turn, to display a closer relationship with the recalcitrant behaviours, ultimately, any one may function as a precursor to any one of the four value eroding behaviours. Moving on to the relationships between the four value-eroding behaviours, several participants argued against the existence of any kind of interrelationship between the constructs while others provided unique examples of instances where a specific primary behaviour could trigger a secondary behaviour.

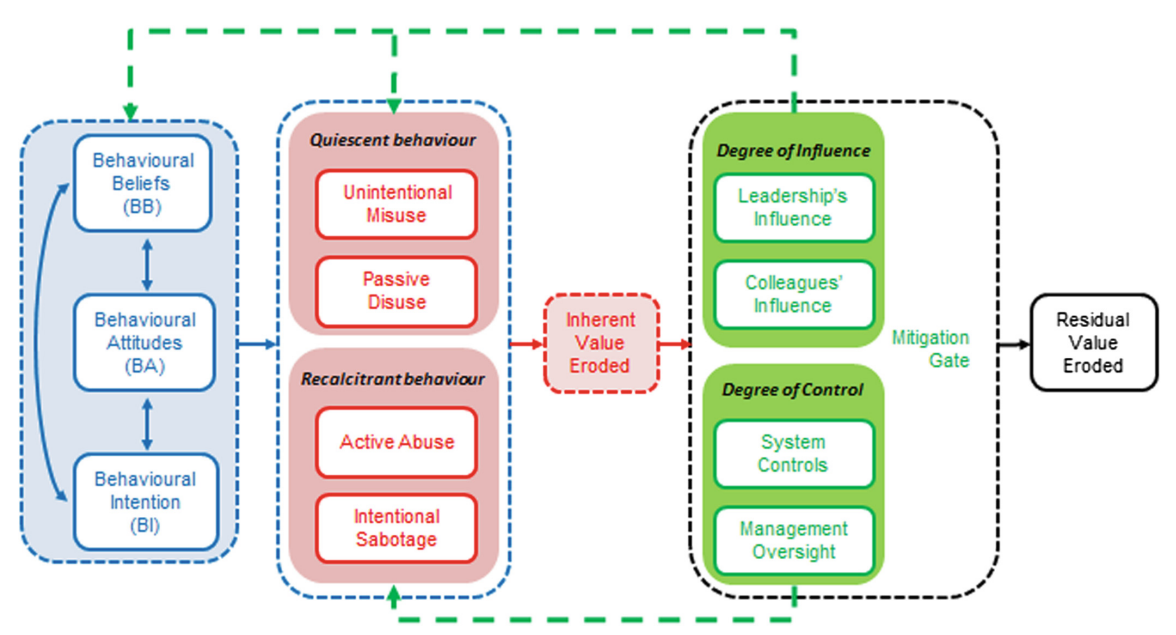

Fig. 2. Adjusted technology value model

While all four the mitigating constructs were perceived to be valid, the two Degree of Control constructs, were seen to be more effective in mitigating value eroding behaviour, while the two Degree of Influence constructs were seen to be less effective yet not as costly in the prevention of behaviours that destroyed business value.

The results from the primary research and ensuing ATVM are consistent with the updated DeLone and McLean Model [15] in that both utilization and user attitudes 
toward technology were shown to be important. The research also supported the Intention to Use construct as it further elucidated the behavioural intent of end-users, occasioning IS abuse. The research furthermore confirmed the problem of increased organisational spent on IT with little realisation or insufficient justification on how, why and when IS investments create business value [16, 17]. In a similar vein, the research supported the literature by explicating the continued challenge that exists within organisations to measure and communicate IT value, noting that while many IT metrics measure performance, they do not measure actual value [18]. The investigation also confirmed the contributions made by several authors maintaining that the primary challenges experienced by technology driven organisations lied with the human element [e.g. 7, 8].

In concluding, the ATVF provides a clear articulation of the actual IS related behavioural activities or actions executed by the human agent contributing directly to business or organisational value erosion and offers a model on how business value can be safeguarded against destructive HCI behaviors. Future studies should focus on refining and validating the proposed ATVM.

\section{References}

1. Silver, M.S., Markus, M.L., Beath, C.M.: The information technology interaction model: a foundation for the MBA core course. MIS Q. 19(3), 361-390 (1995). https://doi.org/10. 2307/249600

2. Bhattacherjee, A.: Understanding information systems continuance: an expectation confirmation model. MIS Q. 25(3), 351-370 (2001). https://doi.org/10.2307/3250921

3. Dawson, G.S., Watson, R.T., Boudreau, M.C.: Information asymmetry in information systems consulting: toward a theory of relationship constraints. J. Manag. Inf. Syst. 27(3), 143-178 (2010). https://doi.org/10.2753/MIS0742-1222270306

4. Linder, S., Foss, N.J.: Agency Theory (No. SMG WP 7/2013) (2013)

5. Davis, E.A.: The effects of technological and organizational innovations on business value in the context of a new product or service deployment. (L. J. S. Calloway Namchul, Ed.) ProQuest Dissertations and Theses. Pace University, United States - New York (2010)

6. Melville, N., Kraemer, K., Gurbaxani, V.: Review: information technology and organizational performance: an integrative model of IT business value. MIS Q. 28(2), 283-322 (2004). https://doi.org/10.2307/25148636

7. Anderson, M.C., Banker, R.D., Ravindran, S.: The new productivity paradox. Commun. ACM 46(3), 91-94 (2003). https://doi.org/10.1145/636772.636776

8. Haspeslagh, P., Noda, T., Boulos, F.: Managing for value: it's not just about the numbers. Harvard Bus. Rev. 79, 65-73 (2001)

9. Goodhue, B.D.L., Thompson, R.L.: Task-technology fit and individual performance. MIS Q. 19(2), 213-236 (1995). https://doi.org/10.2307/249689

10. Tétard, F., Collan, M.: Lazy user theory: a dynamic model to understand user selection of products and services. In: Proceedings of the 42nd Hawaii International Conference on System Sciences (2009)

11. Gurbaxani, V., Whang, S.: The impact of information systems on organizations and markets. Commun. ACM 34(1), 59-73 (1991). https://doi.org/10.1145/99977.99990

12. Flood, R.L.: The relationship of 'systems thinking' to action research. Syst. Pract. Action Res. 23(4), 269-284 (2010) 
13. Wixom, B.H., Todd, P.A.: A theoretical integration of user satisfaction and technology acceptance. Inf. Syst. Res. 16(1), 85-102 (2005). https://doi.org/10.1287/isre.1050.0042

14. Oates, B.J.: Researching Information Systems and Computing. Sage Publications, London (2006)

15. Urbach, N., Müller, B.: The updated DeLone and McLean model of information systems success. In: Dwivedi, Y.K., Wade, M.R., Schneberger, S.L. (eds.) Information Systems Theory: Explaining and Predicting Our Digital Society, vol. 28, pp. 1-18. Springer, New York (2012). https://doi.org/10.1007/978-1-4419-6108-2_1

16. Soh, C., Markus, M.: How IT creates business value: a process theory synthesis. In: ICIS (1995)

17. Schryen, G.: Revisiting IS business value research: what we already know, what we still need to know, and how we can get there. Eur. J. Inf. Syst. 22, 139-169 (2013). https://doi. org/10.1057/ejis.2012.45

18. Mitra, S., Sambamurthy, V., Westerman, G.: Measuring IT performance and communicating value. MIS Q. Exec. 10(1), 47-60 (2011) 